\title{
Diffusion Bonding of Gold
}

\author{
Giles Humpston and Sara J Baker \\ Marconi Materials Technology Ltd, Caswell, Towcester, Northamptonshire NN12 8EQ,UK
}

\begin{abstract}
Gold has a low modulus and does not form an oxide skin when heated in the air. Added to this is its rapid self-diffusion characteristic and these three properties together make this metal suitable for joining gold to gold. This paper presents a summary of a practical diffusion-bonding method for making such joints.
\end{abstract}

Diffusion bonding is a well established method for permanently joining together two metal components. The process basically entails placing the two components in contact and using temperature and time to remove asperities from the interface by vacancy diffusion. The result is a fully dense joint line. Pressure is usually applied perpendicular to the bond region during the heat-treatment in order to force the mating surfaces into the closest possible contact and thereby shorten the process time. Obviously for the process to work it is essential that the metal surfaces are scrupulously clean and free of any oxide or other nonmetallic films.

Among the many attractive features of gold are its low modulus, rapid self-diffusion and absence of an oxide skin, even when heated in air. These three characteristics render it a most suitable metal for effecting diffusion bonded joints. However a recent literature search failed to locate comprehensive data on suitable combinations of temperature, time and pressure to use. Accordingly a limited programme of experimental work was undertaken with this objective in mind.

Because diffusion bonding only involves closure of a joint gap, it is not necessary to employ substantial gold blocks as test pieces. Instead, substrates comprising ordinary borosilicate glass microscope slides were used. These are extremely flat, reasonably tough and will accept a gold coating over an active seed metal. Sputter deposition was chosen to metallize the glass slides because this method is a high energy process and thereby yields exceedingly well adhered metal layers of essentially theoretical density (1). In this instance the glass was sputter-coated with a thin layer of titanium $(0.1 \mu \mathrm{m})$ and platinum $(0.1 \mu \mathrm{m})$ overlaid with $3 \mu \mathrm{m}$ of gold. These metals were deposited in an argon atmosphere of 7 mtorr. The titanium was deposited at 200W target power, $20 \mathrm{~W}$ substrate power, giving a bias of approximately 95 volts. The platinum and gold were both deposited at $100 \mathrm{~W}$ target power. The resulting gold surface was extremely smooth with an Ra of $0.05 \mu \mathrm{m}$.

Square coupons of varying dimensions were diced from the metallized glass slides and subjected to a range of applied temperatures and pressures. The process time was arbitrarily fixed at one hour. After diffusion bonding, a metal stud was attached to each side of the glass sandwich using a high strength epoxy adhesive. The assemblies were then subjected to uniaxial tension until failure at a cross head velocity of $3 \mathrm{~mm}$ per minute.

The results are presented in Figure 1. The graph shows the expected inverse relationship between process temperature and pressure. The line denotes the boundary between good and failed bonds. In the interests of clarity only those results that straddle this line are included in Figure 1. As can be seen, the differentiation between a good and failed bond is quite abrupt and an increase in process temperature of only a few per cent is necessary to go from joints of negligible strength to ones where the epoxy adhesive fails at approximately $10-25 \mathrm{MPa}$. The data are consistent with the limited process conditions reported in the literature $(2,3)$.

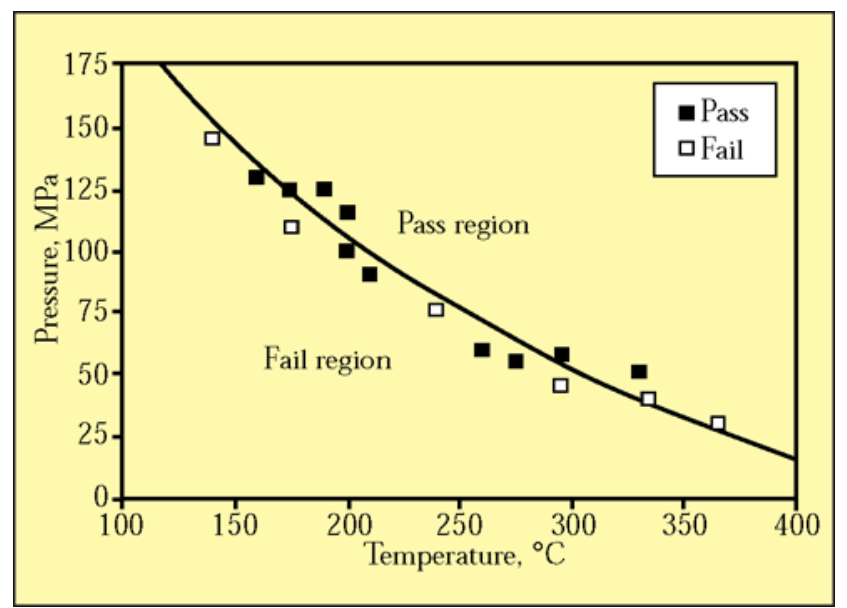

Figure 1 Plot of process temperature against process pressure 
The line on the graph is the fitted curve of a simple natural logarithm equation. Because the correlation coefficient is high it is possible to predict that a successful bond will be achieved without pressure at temperatures above about $430^{\circ} \mathrm{C}$. Certainly it is the author's experience that heattreatment of a bundle of pure gold wires for one hour at $500^{\circ} \mathrm{C}$ resulted in a single rod and some hindsight into gold diffusion bonding!

\section{REFERENCES}

1 P. J. Martin, Gold Bull., 1986, 19(4), 102-116

2 D.L. Ornellas and E. Catalano, Rev. Sci. Instr., 1974, 45(7), 955

3 R.L. Williams and J.B. Tyra, Hughes Aircraft Company, WO Patent 94/17551

\title{
Low Resistance Gold Contacts for Gallium Nitride
}

\author{
Peter Boswell \\ Bricad Associates sarl, route de Genève, 7, CH-1291 Commugny, Switzerland
}

\begin{abstract}
Gold is playing an important role in the development of reliable contacts with a very low contact resistance to gallium nitride.
\end{abstract}

Owing to its chemical inertness, gold is often the ideal contact material for semiconductor devices. However, new generations of semiconductors are continually entering mainstream applications, so device manufacturers must tackle fundamental challenges when continuing to use gold in novel types of contacts. The identification of a viable contact for gallium nitride demonstrates that gold's versatility often lies at the heart of modern contact systems based on sophisticated physical concepts. The system comprises a series of thin films deposited successively on the gallium nitride where the final, relatively thick $(50 \mathrm{~nm})$ film of gold provides the low-resistance connection to the exterior.

Gallium nitride has emerged mainly because the capacities of optical storage media used in domestic audio and video equipment and in computer storage devices increase remorselessly in the quest for higher performance. Solid-state lasers working in the blue to ultraviolet are necessary, and materials based on the II-VI and III-V elements race to become the dominant system. The ZnSe II-VI compound has received the most attention, highlighted by the announcement in 1994 of lasers capable of operating for over 100 hours. However, the GaN III-V compound with superior electronic and physical properties is the material of choice. For instance, the large energy difference for electrons sitting in the conduction and valence bands leads to a small leakage current and a large breakdown voltage, important properties for devices operating at high temperatures and at high frequencies. Unfortunately, producing defect-free $\mathrm{GaN}$ crystals is extremely difficult so there is no commercial source of $\mathrm{GaN}$ substrates for growing the layer structures used in solid-state lasers.

Meanwhile, GaN grown on sapphire substrates is now the material of choice for manufacturing the lasers' cousins, namely light emitting diodes (LEDs) that operate in the blue. So the installed production capacity for high-brightness GaN LEDs has leapt from almost zero a few years ago to over 10 million units per month worldwide. This has paved the way for using GaN LEDs in ubiquitous applications such as traffic lights. The potential for high-temperature and highfrequency applications and for short-wavelength detectors persists, with overall success ultimately depending on the future development of bulk materials and layer growth processes, and on substrate technologies in general. 